\title{
Taper array in silica glass for beam splitting
}

\author{
Zehua Xia ${ }^{1}$, Yan $\mathrm{Li}^{1, *}$, Yanhua Han ${ }^{1}$, Meng $\mathrm{Ge}^{1}$, Jingfu Ye ${ }^{1}$, Duo Deng ${ }^{1}$, Benyang $\mathrm{Wang}^{1}$, Jianmin $\mathrm{Gao}^{2}$, \\ and Shiliang $\mathrm{Qu}^{1}$ \\ ${ }^{1}$ Department of Optoelectronics Science, Harbin Institute of Technology at Weihai, Weihai, 264209, \\ China \\ ${ }^{2}$ School of Energy Science and Engineering, Harbin Institute of Technology, Harbin, 150001, China \\ *Corresponding author: yanli@hitwh.edu.cn
}

\begin{abstract}
We proposed taper array in silica glass for beam splitting which was fabricated by water-assisted femtosecond laser direct writing technology and the subsequent heat treatment. We divided the array into many fabricating cells which were executed automatically in sequences as specified by the program that contained the information for the three-dimensional stage movements. Each cell could fabricated a rectangular cylinder. The size and distribution of the rectangular cylinder could be controlled by adjusting the position of the fabricating cells. Then the heat treatment should be used to reshape the rectangular cylinders into taper array. The experimental results show that the taper periodic microstructures in silica glass are uniform and smooth, and the tapers can divide the incident light into beam array. The results demonstrated that the combination of the water-assisted femtosecond laser direct writing technology and the heat treatment is accessible and practical for the high quality micro-optical elements. These micro-optical elements will have potential applications in fluorescence detection and beam splitter.
\end{abstract}

Keywords: Taper array; Femtosecond laser direct writing; Beam splitter

PACS: 52.38.Mf, 42.79.Fm

\section{Introduction}

In recent years, integrated optical devices have attracted numerous researchers' attention. The devices like beam splitters ${ }^{[1]}$, couplers $^{[2]}$, and modulators ${ }^{[3]}$ as well as the active devices like lasers based on waveguides structures ${ }^{[1,4]}$. At present, the laser direct writing technology is practical and flexible compared with the other fabrication methods, and this technology could realize three-dimensional microstructures in most materials ${ }^{[5,6]}$. In silica glass, the three- 
dimensional hollow microstructures also can be fabricated by femtosecond laser direct writing technology and followed chemical etching ${ }^{[5,7,8]}$. The fabrication of hollow microstructures is relevant to chemical and biological analysis ${ }^{[9]}$. The typical fabrication methods of hollow microstructures like microchannels are soft lithography ${ }^{[10]}$, wet etching ${ }^{[11]}$ and the bonding technology ${ }^{[12]}$. Generally, it is difficult to realized uniform diameter at the opening and middle $\operatorname{area}^{[8]}$. It is more difficult to fabricate uniform periodic microstructures in microchannel by femtosecond laser direct writing technology followed chemical etching. As the 3D microchip can be realized by water-assisted femtosecond laser direct writing technology ${ }^{[13]}$. Using this method, the hollow and periodic microstructures in silica glass can be fabricated directly.

In this paper, we present a method for fabricating periodic microstructures in silica glass using water-assisted femtosecond laser direct writing technology as the beam splitter. Using this method, the periodic microstructures could be located in any place, without limitation of space structure and fabricating length. The distribution of the rectangular cylinders should be designed and fabricated automatically by three-dimensional stage (Prior Scientific Inc.), cell by cell. After subsequent heat treatment, the internal surface of fabricating area could be much smoother which was crucial for optical elements. Finally, the rectangular cylinders array could be reshaped and the taper array was realized in silica glass. The experimental results confirmed that taper microstructures have beam splitting capacity. This taper beam splitter has potential application in various fields, such as biomedicine, fluorescence detection, and beam splitters.

\section{Fabrication of taper array}

In the experiments, Ti: sapphire regenerative amplified laser system (Coherent Inc.) was used as the femtosecond laser source. The repetition rate, central wavelength and pulse width of the femtosecond laser are $1 \mathrm{kHz}, 800 \mathrm{~nm}$, and 120fs, respectively. For selecting the high quality laser beam a circular aperture was employed with the diameter of $5 \mathrm{~mm}$. The periodic microstructures were fabricated automatically, so a mechanical shutter with $100 \mathrm{~ms}$ response time should be used to select the laser direct writing area. The experiments were carried out by using water-assisted femtosecond laser direct writing technology and the laser beam was focused into the distilled water by an optical microscope (20×/0.45, Nikon). The used base material of sample was commercially available silica glass. A computer controlled three-dimensional stage (Prior Scientific Inc.) with the resolution $100 \mathrm{~nm}$ was used to move the glass sample. The whole 
experimental process was monitored by a charge coupled device (CCD) in real time. The experimental setup is shown in Figure $1^{[13]}$.

The silica glass was fasten on the three-dimensional stage and a rubber hose was fixed under the silica glass. The laser beam was focused in the rubber hose for inducing the breakdown in the distilled water ${ }^{[14]}$. We first drilled a hole from the bottom of the silica glass, then started the programme for fabricating the periodic microstructures automatically. When the laser beam ablated the silica glass, the silica material could be etched into debris which dispersed into the distilled water. Therefore, the hollow microstructure could be fabricated. With the length increasing of the hollow structure, the distilled water was pumped into the ablated area for cleaning the high level debris through the rubber hose. Therefore the effects of blocking and redeposition generated by the ablated debris are greatly reduced. The use of the rubber hose ensured the effectively cleaning and the continuously pumping fresh water into the ablating area. Therefore, the fabricating scale could be increased and the periodic microstructures could be fabricated in one step.

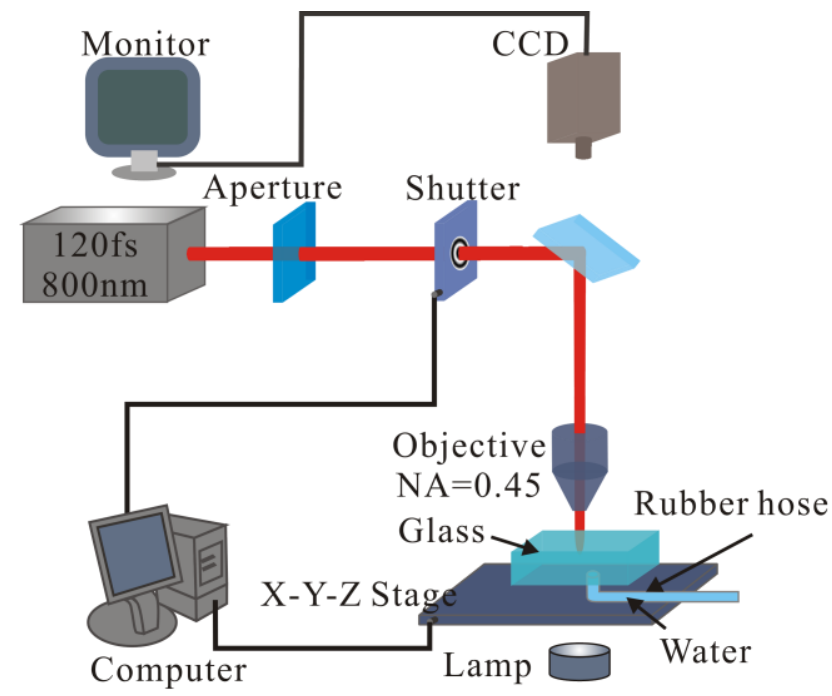

Figure 1. The experimental setup for fabrication of the microchannels

In order to ablate the silica glass into debris as much as possible and improve the fabricating efficiency, the repetition rate of the femtosecond laser was set $1 \mathrm{kHz}$ and the scanning speed was set $260 \mu \mathrm{m} / \mathrm{s}$. The femtosecond laser was focused in the distilled water by a $20 \mathrm{X}$ objective $(\mathrm{NA}=0.45)$ for fabricating the periodic microstructures. Two patterns of the array were designed as shown in Figure 2(a), square pattern in the left and triangle pattern in the right. From Figure 
2(a), it can be seen that, the interval of adjacent rectangular cylinders is $50 \mu \mathrm{m}$, both horizontally and vertically. The whole fabricating process was executed automatically in sequences as specified by the program that contained the information for the three-dimensional stage movements. The pattern of the array is divided into many fabricating cells, each cell is a rectangular ring with the length and width of $90 \mu \mathrm{m}$ and $80 \mu \mathrm{m}$ respectively, as shown in the center of the Figure 2(a). The central region of the fabricating cell could not be ablated by the femtosecond laser, which is a rectangle with $20 \mu \mathrm{m}$ long and $10 \mu \mathrm{m}$ wide. Therefore, the rectangular cylinders were fabricated cell by cell along vertical or horizontal direction. Also the square and triangle pattern could be fabricated by adjusting the interval of the fabricating cells. In the whole fabricating process, the ablated debris should be remained at a relatively low level in the focused region for avoiding the strong scattering of the femtosecond laser. So the distilled water was pumped into the focused region continuously through the rubber hose for cleaning the hollow microstructures. Effective cleaning process could be the key to realize the array.

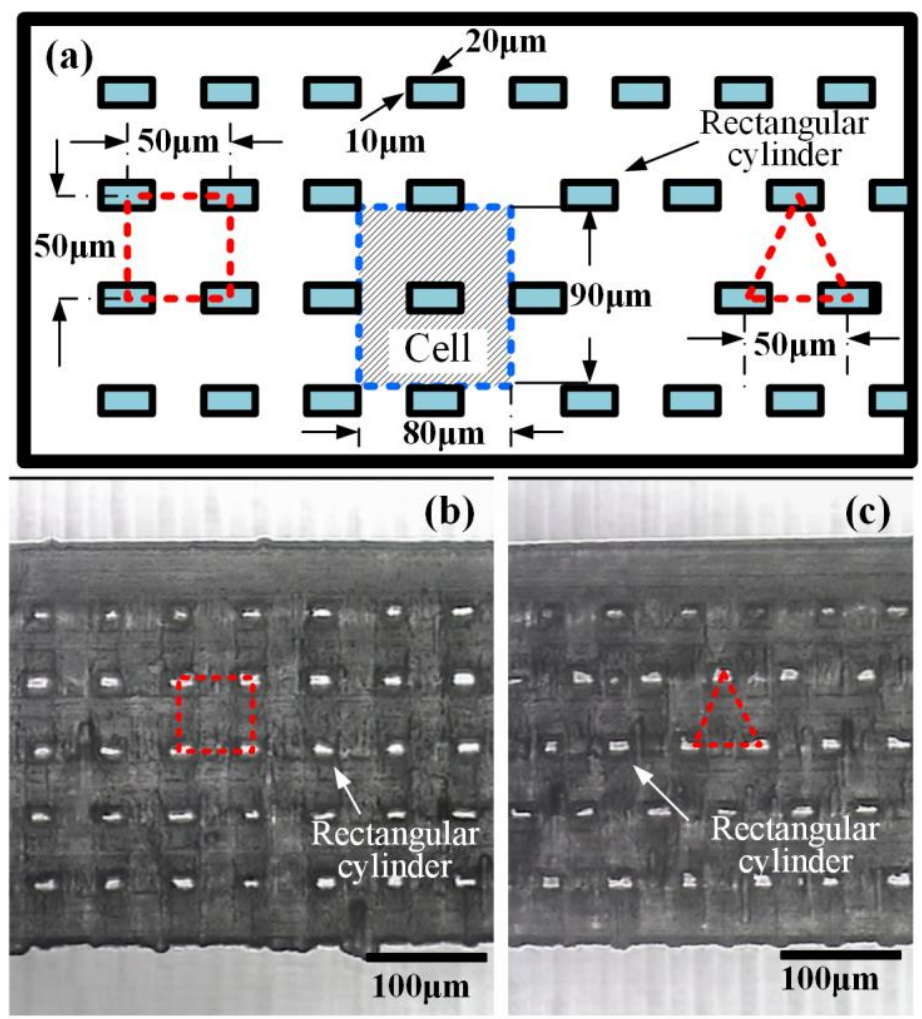

Figure 2. (a) The schematic of the designed pattern, (b) the fabricated array with square distribution, (c) the fabricated array with triangle distribution. 
In this experiments, the used energy of the femtosecond laser pulses was $6 \mu \mathrm{J}$ and the repetition rate was $1 \mathrm{kHz}$. And, the fabricating cell should be scanned by a constant speed $260 \mu \mathrm{m} / \mathrm{s}$. When the microstructures were realized, it was necessary to pump more distilled water to eject the debris. Then, the sample was put into an ultrasonic cleaner to remove the redeposited debris. The fabricated arrays are shown in Figure 2(b) and Figure 2(c) with square and triangular distribution. From Figure 2(b) and Figure 2(c) we can see that the ablated region is rough and the rectangular cylinders are not regular. Because of the water-assisted femtosecond laser direct writing technology, the silica glass was etched into debris by the high focused femtosecond laser. The rough internal surface is inevitable which could lead to a reduction of light transmittance. So we have to smooth the internal surface and reshape the rectangular cylinders into tapers via heat treatment.

\section{Results and Discussion}

In order to form the taper array, heat treatment should be used and simultaneously the internal surface could be improved. The silica sample was put into a tube furnace, when the temperature rose to $750^{\circ} \mathrm{C}$, then the power of the tube furnace was turned off, where the sample was cooled naturally. After heat treatment, the ablated region is bright and smooth as seen in Figure 3 (a). Because of the heat treatment, the rough internal surface including the surface of the rectangular cylinders were melted, under effect of surface tension the rectangular cylinders became tapers. Top view of the taper is oval with major axis diameter and minor axis $33 \mu \mathrm{m}$ and $16 \mu \mathrm{m}$, respectively. Comparing to the rectangle (top view of the rectangular cylinders with $10 \mu \mathrm{m}$ width and $20 \mu \mathrm{m}$ length), the lengths have increased by $60 \%$. After heat treatment the edge distance between adjacent taper became small, but we can see that the distance of the centers

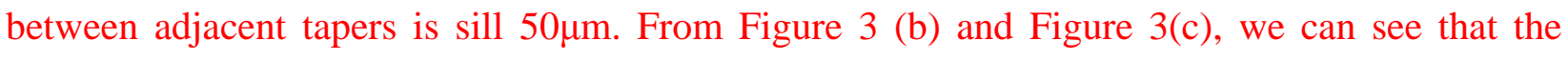
distribution of the fabricated tapers are still square and triangle. In order to know the profile of the tapers we fabricated, we investigated the cross-section of the array. After the polishing process, we obtained the image of the tapers through the optical microscope and the CCD camera, as shown in Figure 3(d). The black regions are the air cavities with circular sections, and the diameters of the circular sections are almost the same. The adjacent bright fields were the shape of fabricated tapers, and obviously the rectangular cylinders become tapers after heat treatment. 


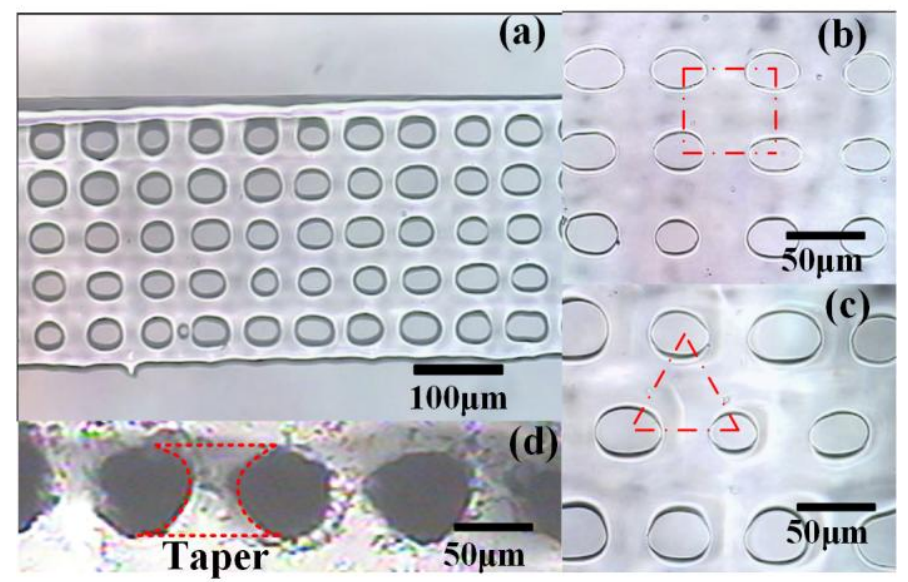

Figure 3. (a) The silica sample after heat treatment. The distribution of the rectangular cylinders (b) square

(c) triangle, (d) The image of the cross-section of the array.

Subsequently, we investigated the taper array as the beam splitter. Figure 4 shows the schematic of the optical transmission performance system. As shown in Figure 4 the He-Ne laser beam was selected by an aperture and focused on the surface of the sample by a lens (L1). Another lens (L2) was used as a collimated lens and the beam array was captured by a CCD camera. Figure 5. (a) shows the result of the rectangular distribution beam array. The total energy of the beam array is $16.3 \%$ of the incident light. The hexagonal distribution beam array is shown in Figure 5. (b), which total energy of the beam array is $13.7 \%$ of the incident light. The images demonstrated that the taper microstructures could divide the incident laser into different beam array and the spots are circular. Moreover, the taper arrays were fabricated in the silica glass by water assisted femtosecond laser direct writing technology which could integrated the microstructures into microfluidic chips or micro imaging system.

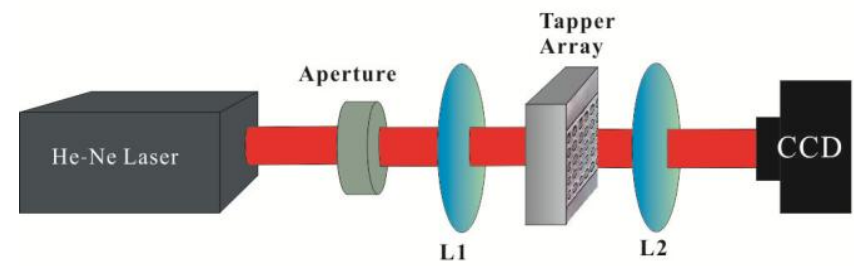

Figure 4. Schematic of the optical transmission performance system. 


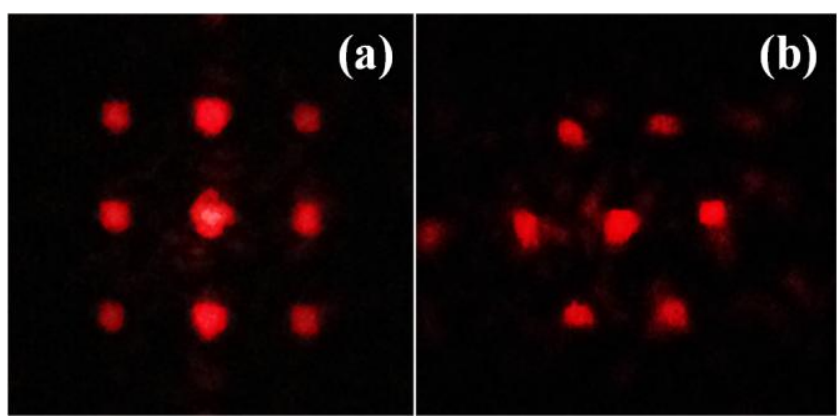

Figure 5. (a) Rectangular beam array (b) hexagonal beam array

\section{Conclusions}

In this paper, we successfully fabricated the taper array as beam splitter in silica glass by using water-assisted femtosecond laser direct writing technology. Heat treatment was used to improve the roughness of the internal surface and reshape the rectangular cylinders into tapers. The distribution of the tapers could be controlled by changing the interval of fabricating cells. Experimental results show that using this fabricating method the 3D periodic microstructures could be realized in silica glass automatically. The taper microstructures array can clearly divide the incident light into beam array. The presented method in this paper is also demonstrated that it's convenient to fabricate 3D microstructures in silica glass, which is of great application prospect in the complex integrated devices.

\section{Acknowledgment}

This work is supported by National Science Foundation of China (Grant Nos. 11304064 and 11304065), the Science and Technology Foundation of Shandong Province (Grant No. ZR2013AQ002), the Science and Technology Development Plan of Weihai (Grant No. 2013DXGJ10), the project (Grant No.201410030-03) supported by the Special Scientific Research Found of Public Service industry of Quality Inspection.

\section{References}

[1] T. Calmano, C. Krankel, and G. Huber, Laser oscillation in Yb:YAG waveguide beam-splitters with variable splitting ratio, Opt. Lett., 40 (2015) 1753-1756.

[2] S. Somekh, E. Garmire, A. Yariv, H. L. Garvin, and R. G. Hunsperger, Channel optical waveguide directional couplers, Applied Physics Letters, 22 (1973) 46-47.

[3] B. A. Block, T. R. Younkin, P. S. Davids, M. R. Reshotko, P. Chang, B. M. Polishak, S. Huang, J. Luo, and A. K. Y. Jen, Electro-optic polymer cladding ring resonator modulators, Opt. Express, 16 (2008) 18326-18333. 
[4] D. Choudhury, J. R. Macdonald, and A. K. Kar, Ultrafast laser inscription: perspectives on future integrated applications, Laser \& Photonics Reviews, 8 (2014) 827-846.

[5] F. He, Y. Cheng, Z. Xu, Y. Liao, J. Xu, H.Sun, C. Wang, Z. Zhou, K. Sugioka, K. Midorikawa, Y. $\mathrm{Xu}$, and $\mathrm{X}$. Chen, Direct fabrication of homogeneous microfluidic channels embedded in fused silica using a femtosecond laser, Opt. Lett., 35 (2010) 282-284.

[6] X. Liu, S. Qu, Y. Tan, and F. Chen, Preservation of fluorescence and Raman gain in the buried channel waveguides in neodymium-doped $\mathrm{KGd}(\mathrm{WO} 4) 2(\mathrm{Nd}: \mathrm{KGW})$ by femtosecond laser writing, Appl. Opt., 50 (2011) 930-934.

[7] V. Maselli, R. Osellame, G. Cerullo, R. Ramponi, P. Laporta, L. Magagnin, and P. L. Cavallotti, Fabrication of long microchannels with circular cross section using astigmatically shaped femtosecond laser pulses and chemical etching, Appl. Phys. Lett., 88 (2006) 191107.

[8] Y. Li and S. Qu, Fabrication of spiral-shaped microfluidic channels in glass by femtosecond laser, Material Letters, 64 (2010) 1427-1429.

[9] K. Sato, M. Tokeshi, T. Kitamori, and T. Sawada, Integration of Flow Injection Analysis and Zeptomole-Level Detection of the Fe(II)-o-Phenanthroline Complex, Anal. Sci., 15 (1999) 641-645.

[10] B. Kavčič, D. Babič, N. Osterman, B. Podobnik, and I. Poberaj, Magnetically actuated microrotors with individual pumping speed and direction control, Appl. Phys. Lett. , 95 (2009) 023504.

[11] C. Hnatovsky, R. S. Taylor, E. Simova, P. P. Rajeev, D. M. Rayner, V. R. Bhardwaj, and P. B. Corkum, Fabrication of microchannels in glass using focused femtosecond laser radiation and selective chemical etching, Appl. Phys. A, 84 (2006) 47-61.

[12] L. Martynova, L. E. Locascio, M. Gaitan, G. W. Kramer, R. G. Christensen, and W. A. MacCrehan, Fabrication of plastic microfluidic channels by imprinting methods, Anal. Chem., 69 (1997) 47834789.

[13] Y. Li and S. Qu, Water-assisted femtosecond laser ablation for fabricating three-dimensional microfluidic chips, Current Applied Physics, 13 (2013) 1292-1295.

[14] Y. Li and S. Qu, Femtosecond laser-induced breakdown in distilled water for fabricating the helical microchannels array, Optics Letters, 36 (2011) 4236-4238. 